\title{
Concurrent Chemoradiotherapy with S-1 Compared with Concurrent Chemoradiotherapy with Docetaxel and Cisplatin for Locally Advanced Esophageal Squamous Cell Carcinoma
}

\author{
Xi-Lei Zhou \\ Huaian First People's Hospital \\ Chang-Hua Yu \\ Huaian First People's Hospital \\ Wan-Wei Wang \\ Huaian First People's Hospital \\ Fu-Zhi Ji \\ Huaian First People's Hospital \\ Yao-Zu Xiong \\ Huaian First People's Hospital \\ Wei-Guo Zhu \\ Huaian First People's Hospital \\ Yu-Suo Tong ( $\nabla$ tongyusuo@163.com ) \\ Huaian First People's Hospital
}

\section{Research}

Keywords: esophageal cancer, chemoradiotherapy, S-1, docetaxel, cisplatin

Posted Date: October 2nd, 2020

DOI: https://doi.org/10.21203/rs.3.rs-84169/v1

License: (c) (i) This work is licensed under a Creative Commons Attribution 4.0 International License.

Read Full License

Version of Record: A version of this preprint was published at Radiation Oncology on May 26th, 2021. See the published version at https://doi.org/10.1186/s13014-021-01821-6. 


\section{Abstract}

Background: This retrospective study was to assess and compare the toxicity and efficacy of concurrent chemoradiotherapy (CCRT) with S-1 or docetaxel and cisplatin in patients with locally advanced esophageal squamous cell carcinoma (ESCC).

Methods: Patients who had locally advanced ESCC and received CCRT with S-1 $\left(70 \mathrm{mg} / \mathrm{m}^{2}\right.$ twice daily on days 1-14, every 3 weeks for 2 cycles, S-1 group) or docetaxel $\left(25 \mathrm{mg} / \mathrm{m}^{2}\right)$ and cisplatin $\left(25 \mathrm{mg} / \mathrm{m}^{2}\right)$ on day 1 weekly (DP group) between 2014 and 2016 were retrospectively analyzed. Treatment-related toxicities, response rate, and survival outcomes were compared between groups.

Results: A total of 175 patients were included in this study (72 in the S-1 group and 103 in the DP group). Baseline characteristics were well balanced between the two groups. The incidence of grade 3-4 adverse events were significantly lower in the S- 1 group than in the DP group $(22.2 \%$ versus $45.6 \%, p=0.002)$. The objective overall response rate (complete response + partial response) was $68.1 \%$ in the S-1 group, and $73.8 \%$ the DP group $(p=0.497)$. The 3 -year overall survival was $34.7 \%$ in the $\mathrm{S}-1$ group, and $38.8 \%$ in the DP group $(p=0.422)$. The 3-year progression free survival in the DP group was higher than that in the S-1 group but without significant difference (33.0\% versus $25.0 \%, p=0.275)$.

Conclusion: CCRT with S-1 is not inferior to CCRT with docetaxel and cisplatin and is better tolerated in in elderly patients with locally advanced ESCC.

\section{Background}

Esophageal squamous cell cancer (ESCC) is fourth leading cause of cancer-related death in China.[1] Approximate $70 \%$ of patients with ESCC have locally advanced disease and overall survival (OS) remains poor.[2] For these patients, concurrent chemoradiotherapy (CCRT) has been regarded as the standard treatment.[3] However, the optimal concurrent chemotherapy regimen for this disease remains controversial. The combination of cisplatin and fluorouracil (PF) is one of the most commonly used concurrent chemotherapy regimens.[4] In RTOG-85-01, concurrent chemotherapy could be administered in only 89 of 130 patients (68\%) because of PF-related toxic effects. Furthermore, even after CCRT, long-term survival remains unsatisfactory, with a 5-year OS of less than 27\%.[5] To improve the survival, many chemotherapeutic agents have been evaluated, such as docetaxel, paclitaxel, and cetuximab.[6] Of them, the combination of docetaxel with cisplatin has shown promising results in patients with locally advanced or recurrent ESCC when combined with radiotherapy (RT).[7, 8] A recent randomized trial demonstrated that this regimen could result in higher overall response rates and longer OS compared with PF-based CCRT with tolerable toxicities in locally advanced ESCC.[9]

S-1, a new biochemical modulator of 5-fluorouracil, is an oral fluorinated pyrimidine formulation that consists of tegafur (a prodrug of 5-FU), gimeracil, and oteracil.[10] The anticancer effect of S-1 has been widely used in gastric cancer, non-small cell lung cancer, head and neck cancer, and pancreatic cancer. [11-14] In addition to cytotoxic activity, S-1 can enhance radiosensitivity in tumor cells by suppressing 


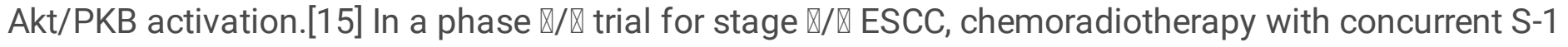
and cisplatin showed highly promising activity with a complete response rate of 59.5\%.[16] Although, a number of studies have demonstrated encouraging results of S-1 in ESCC treatment, no study has investigated the safety and efficacy of docetaxel plus cisplatin versus S-1 concurrent with RT in patients with ESCC.

The aim of this study was to compare the differences in treatment toxicity and efficacy between S-1 and docetaxel with cisplatin as CCRT in patients with locally advanced ESCC.

\section{Methods}

\section{Patients}

This study was approved by the institutional review board of Huai'an First Hospital. Informed consent was exempted due to the retrospective nature of the study. Between January 2014 and December 2016, patients with locally advanced ESCC who received CCRT with S-1 or docetaxel and cisplatin at our institute were retrospectively analyzed. Inclusion criteria were histologically confirmed ESCC, Karnofsky performance status (KPS) score $\geq 70$, less than 75 years old, no significant medical disease. Patients with history of previous or concomitant malignancy were excluded as well as cases with missing relevant staging information or inadequate follow up. Clinical staging (UICC, 6th edition) evaluations were based on esophagogram and enhanced CT scan of neck, chest and upper abdomen. Clinical data collected from each patient included age, gender, KPS score, primary tumor location, clinical stage, radiation dose, and tumor response to treatment. Blood samples at baseline, during (weekly) and 4 weeks after CCRT were also collected for evaluation haemoglobin concentration, neutrophil counts and platelet counts.

\section{Chemotherapy regimens}

Chemotherapy began on day 1, concurrent with the beginning of RT.

S-1 group: S-1 (70 mg/m²) was given orally twice daily on days $1-14$ every 3 weeks for 2 cycles. After CCRT, patients received two cycles of consolidation chemotherapy with S-1 at the same dose levels as during CCRT every 3 weeks.

Docetaxel + cisplatin (DP) group: The DP regimen comprised 5 or 6 cycles of docetaxel $\left(25 \mathrm{mg} / \mathrm{m}^{2}\right.$ per day) and cisplatin ( $25 \mathrm{mg} / \mathrm{m}^{2}$ per day) during RT at day $1,8,15,22,29$, and 36 . Four weeks after completion of CCRT, patients received 2 cycles of consolidation chemotherapy (docetaxel $75 \mathrm{mg} / \mathrm{m}^{2}$ on day 1 , cisplatin $25 \mathrm{mg} / \mathrm{m}^{2}$ on days $1-3$ ) with a 4-week interval.

\section{Radiotherapy}


All of the patients underwent three-dimensional conformal radiotherapy (3D-CRT) or intensity modulated radiotherapy (IMRT) via a $6 \mathrm{MV}$ X-ray beam. A total radiation dose of 50-60 Gy (median dose 50.4 Gy) was given in daily fractions of 1.8-2Gy. The clinical target volume (CTV) included the gross tumor volume (GTV) with 3-cm craniocaudal margin, the metastatic lymph nodes, and regional lymph node. We have previously reported the details of regional lymph node for tumor in different locations at our institute.[17]

\section{Toxicity and response assessment}

Toxicities were graded according to the National Institute Common Terminology Criteria for Adverse Events (NCI CTCAE) version 4.0. Acute treatment-related toxicity was defined as adverse events that occurred between the beginning of treatment and 3 months after CCRT.

The treatment response was evaluated using the Response Evaluation Criteria in Solid Tumors (RECIST) with esophagography and chest CT scan 4-6 weeks after completion of CCRT.[18] The objective response rate (ORR) was defined as complete response (CR) + partial response (PR).

\section{Follow-up evaluation}

The first follow-up evaluation was performed 4- 6 weeks after completion of CCRT, followed by every 6 months for the next 2 year, and then every year or until death. Follow-up data were obtained from patient medical records, referring physicians, and telephone interview. Each visit included history, physical examination, esophagogram and enhanced CT scan of neck, chest and upper abdomen. We evaluated post-treatment recurrence on esophagogram and CT scan and compared these data with original CTbased radiation treatment plan.

\section{Statistical analysis}

Patient characteristics and treatment-related toxicity rates were compared using Chi-square test or Fisher's exact test. OS was calculated from the date of diagnosis to death or last follow-up. Data from patients that had not died by the time of analysis were censored. Progression free survival (PFS) was defined as the time from diagnosis to first recurrence or metastasis, or last follow-up. Data from patients that were alive without tumor progression at the time of analysis were censored. Survival curves are analyzed by Kaplan-Meier method. All analyses were performed using SPSS 20.0. A two-side $p$ value less than 0.05 was considered statistically significance.

\section{Results}

\section{Patient and treatment characteristics}


Patient and treatment characteristics between the two groups are shown in Table 1. A total of 175 patients (72 in the S-1 group and 103 in the DP group) were eventually included in this study. The baseline patient and tumor characteristics (location, tumor length, and clinical stage) were well balanced between groups. The median age was 64 years (range $47-74$ years) in the S-1 group and 62 years (range 49-74 years) in the DP group. Among all of these patients, tumor invasion to adjacent organs was seen in 80 patients $(45.7 \%)$ and lymph node metastasis in 125 patients $(71.4 \%)$. 
Table 1

Baseline clinical characteristics

\begin{tabular}{|c|c|c|c|}
\hline Patient characteristic & $\begin{array}{l}\text { DP group } \\
(n=103)\end{array}$ & $\begin{array}{l}\text { S-1 group } \\
(n=72)\end{array}$ & $p$ value \\
\hline Age (median, year) & 62 & 64 & 0.647 \\
\hline$<60$ & $48(46.6 \%)$ & $31(43.1 \%)$ & \\
\hline$\geq 60$ & $55(53.4 \%)$ & $41(56.9 \%)$ & \\
\hline Gender & & & 0.873 \\
\hline Male & $65(63.1 \%)$ & 47 (65.3\%) & \\
\hline Female & $38(36.9 \%)$ & $25(34.7 \%)$ & \\
\hline KPS & & & 0.756 \\
\hline 90 & $43(41.7 \%)$ & $28(38.9 \%)$ & \\
\hline$\geq 70$ & $60(58.3 \%)$ & $44(61.1 \%)$ & \\
\hline Tumor location & & & 0.394 \\
\hline Upper third & $28(27.2 \%)$ & $16(22.2 \%)$ & \\
\hline Middle third & $60(58.3 \%)$ & $40(55.6 \%)$ & \\
\hline Lower third & $15(14.5 \%)$ & $16(22.2 \%)$ & \\
\hline Primary tumor length (cm) & & & 0.759 \\
\hline$<5$ & $56(54.4 \%)$ & $41(56.9 \%)$ & \\
\hline$\geq 5$ & $47(45.6 \%)$ & $31(43.1 \%)$ & \\
\hline Smoking & & & 0.273 \\
\hline Nonsmoker & $43(41.7 \%)$ & $24(33.3 \%)$ & \\
\hline Smoker & $60(58.3 \%)$ & $48(66.7 \%)$ & \\
\hline Drinking & & & 0.358 \\
\hline No & $49(47.6 \%)$ & $29(40.3 \%)$ & \\
\hline Yes & $54(52.4 \%)$ & $43(59.7 \%)$ & \\
\hline Differentiation & & & 0.838 \\
\hline Well & $14(13.6 \%)$ & $8(11.1 \%)$ & \\
\hline
\end{tabular}

Abbreviations: KPS: Karnofsky performance status, 3D-CRT: three-dimensional conformal radiotherapy, IMRT: intensity modulated radiotherapy. 


\begin{tabular}{|c|c|c|c|}
\hline Patient characteristic & $\begin{array}{l}\text { DP group } \\
(n=103)\end{array}$ & $\begin{array}{l}\text { S-1 group } \\
(n=72)\end{array}$ & $p$ value \\
\hline Moderate & $59(57.3 \%)$ & $44(61.1 \%)$ & \\
\hline Poor & $30(29.1 \%)$ & $20(27.7 \%)$ & \\
\hline Clinical T stage & & & 1.000 \\
\hline No invasion to adjacent organs & $56(54.4 \%)$ & $39(54.2 \%)$ & \\
\hline Invasion to adjacent organs & $47(45.6 \%)$ & $33(45.8 \%)$ & \\
\hline Clinical N stage & & & 0.867 \\
\hline NO & $30(29.1 \%)$ & $20(27.8 \%)$ & \\
\hline N1 & $73(70.9 \%)$ & $52(72.2 \%)$ & \\
\hline Reason for no surgery & & & 0.924 \\
\hline Patient refusal & $8(7.7 \%)$ & $6(8.3 \%)$ & \\
\hline Surgical contraindication & $15(14.6 \%)$ & $9(12.5 \%)$ & \\
\hline Unresectable disease & $65(63.1 \%)$ & $44(61.1 \%)$ & \\
\hline Unknown & $15(14.6 \%)$ & $13(18.1 \%)$ & \\
\hline Radiation dose (Gy) & & & 0.698 \\
\hline 50 & $34(33.0 \%)$ & $23(31.9 \%)$ & \\
\hline 50.4 & $46(44.7 \%)$ & $29(40.3 \%)$ & \\
\hline$>50.4$ & $23(22.3 \%)$ & $20(27.8 \%)$ & \\
\hline Radiotherapy techniques & & & 0.410 \\
\hline 3D-CRT & $30(29.1 \%)$ & $26(36.1 \%)$ & \\
\hline IMRT & $73(70.9 \%)$ & $46(63.9 \%)$ & \\
\hline Consolidation chemotherapy & & & 0.537 \\
\hline Yes & $61(59.2 \%)$ & $39(54.2 \%)$ & \\
\hline No & $42(40.8 \%)$ & $33(45.8 \%)$ & \\
\hline
\end{tabular}

Of the treatment characteristics, most patients received a radiation dose of 50-50.4 Gy in both groups, and the difference was not significant between the two groups $(p=0.698)$. Sixty-two patients $(86.1 \%)$ in 
the S-1 group completed the planned 2 cycles of S-1 based concurrent chemotherapy, while 73 patients $(70.9 \%)$ in the DP group completed the all cycles of concurrent chemotherapy $(p=0.027)$. Planned RT was completed in 68 patients (94.4\%) in S-1 group, and 94 patients (91.3\%) in the DP group. In the S-1 group, 39 patients $(54.2 \%)$ received 2 additional cycles of consolidation chemotherapy after CCRT, as did 61 patients $(59.2 \%)$ in the DP group $(p=0.537)$.

\section{Treatment-related toxicities and mortality}

Acute treatment-related toxicities are shown in Table 2. The incidence of grade 3-4 adverse events were significantly lower in the S-1 group than in the DP group $(22.2 \%$ versus $45.6 \%, p=0.002)$. In the S-1 group, 13 patients $(18.1 \%)$ experienced a grade 3 toxicity and $3(4.1 \%)$ a grade 4 toxicity. However, in the DP group, 31 patients (30.1\%) experienced a grade 3 toxicity and $16(15.5 \%)$ a grade 4 toxicity. In terms of hematological toxicities, grade 3-4 leukopenia and neutropenia were more frequently observed in the DP group than in the S-1 group (leukopenia: $34.0 \%$ versus $12.5 \%, p=0.001$, neutropenia: $29.1 \%$ versus $9.7 \%$, $p=0.002$ ). There were no statistical differences in the incidence of grade 3-4 non-hematological toxicities during CCRT between the two groups, with the exception of nausea/vomiting $(9.7 \%$ versus $1.4 \%$, $p=0.028$ ), which was more frequent in the DP group. There were 2 treatment-related deaths in the DP group. One patient died due to aspiration pneumonia. The other patient died due to trachea-esophageal fistula. No treatment-related death was observed in the S-1 group. 
Table 2

Treatment-related toxicities during CCRT

\begin{tabular}{|llll|}
\hline Toxicities & $\begin{array}{l}\text { DP group } \\
(\mathbf{n}=103)\end{array}$ & $\begin{array}{l}\text { S-1 group } \\
(\mathbf{n}=72)\end{array}$ & $p$ value \\
\hline Overall toxicity $\geq 3$ & $47(45.6 \%)$ & $16(22.2 \%)$ & 0.002 \\
\hline Grade 3 & $31(30.1 \%)$ & $13(18.1 \%)$ & 0.079 \\
\hline Grade 4 & $16(15.5 \%)$ & $3(4.1 \%)$ & 0.024 \\
\hline Hematological toxicities $\geq 3$ & & & \\
\hline Anemia & $7(6.8 \%)$ & $4(5.6 \%)$ & 0.767 \\
\hline Leukopenia & $35(34.0 \%)$ & $9(12.5 \%)$ & 0.001 \\
\hline Neutropenia & $30(29.1 \%)$ & $7(9.7 \%)$ & 0.002 \\
\hline Thrombocytopenia & $6(5.8 \%)$ & $2(2.8 \%)$ & 0.473 \\
\hline Non-hematological toxicities $\geq 3$ & & & \\
\hline Esophagitis & $24(23.3 \%)$ & $12(16.7 \%)$ & 0.344 \\
\hline Nausea/vomiting & $10(9.7 \%)$ & $1(1.4 \%)$ & 0.028 \\
\hline Mucositis & $4(3.9 \%)$ & $3(4.1 \%)$ & 1.000 \\
\hline Fatigue & $9(8.7 \%)$ & $5(6.9 \%)$ & 0.781 \\
\hline Pneumonitis & $5(4.9 \%)$ & $3(4.1 \%)$ & 1.000 \\
\hline
\end{tabular}

Next, we further investigated the adverse effects in elderly patients. A total of 96 patients were older than 60 years (41 patients in the S-1 group and 55 in the DP group). In the S-1 group, the rate of $\geq$ grade 3 adverse effects was $24.3 \%$ (10/41) for patients $\geq 60$ years and $19.4 \%(6 / 31)$ for those $<60$ years $(p=$ $0.776)$. However, in the DP group, the rate of $\geq$ grade 3 adverse effects was $58.1 \%(32 / 55)$ for patients $\geq$ 60 years, which was significantly higher $(31.3 \%, 15 / 48)$ than those $<60$ years $(p=0.01)$.

\section{Response to treatment}

Tumor response is shown in Table 3. Of the 72 patients in the S-1 group, CR and PR were achieved in 18 patients (25.0\%) and 31 patients (43.1\%), respectively. In the DP group, 29 patients (28.2\%) had a CR and 47 patients $(45.6 \%)$ had a PR. The ORR was similar in the S- 1 group and the DP group $(68.1 \%$ versus $73.8 \%, p=0.497)$. 
Table 3

Tumor response after treatment

\begin{tabular}{|llll|}
\hline Response & $\begin{array}{l}\text { DP group } \\
\mathbf{n}=103\end{array}$ & $\begin{array}{l}\text { S-1 group } \\
\mathbf{n}=\mathbf{7 2}\end{array}$ & p value \\
\hline CR & $29(28.2 \%)$ & $18(25.0 \%)$ & \\
\hline PR & $47(45.6 \%)$ & $31(43.1 \%)$ & \\
\hline SD & $25(24.3 \%)$ & $21(29.1 \%)$ & 0.497 \\
\hline PD & $2(1.9 \%)$ & $2(2.8 \%)$ & \\
\hline ORR & $76(73.8 \%)$ & $49(68.1 \%)$ & \\
\hline $\begin{array}{l}\text { Abbreviations: CR: complete response, PR: partial response, SD: stable disease, PD: progressive } \\
\text { disease, ORR: objective response rate. }\end{array}$ & \\
\hline
\end{tabular}

\section{Follow up and survival}

During the follow-up period, 57 patients (79.2\%) in the S-1 group had disease progression, including 15 patients in primary tumor, 25 in regional lymph node, and 17 in distant metastasis. For patients in the DP group, disease progression was observed in 75 patients (72.8\%). The initial sites of tumor progression were detailed as follows: primary tumor in 19 patients, regional lymph node in 35 patients, and distant metastasis in 21 patients. The 1- and 3-year PFS rates were $65.3 \%$ and $25.0 \%$, respectively in the S-1 group, and $72.8 \%$ and $33.0 \%$, respectively in the DP group. PFS was longer in the DP group than that in the S-1 group but without significant differences (Fig. 1A, $p=0.275)$. Of note, $42.1 \%(24 / 57)$ and $32.0 \%$ $(24 / 75)$ of patients who had disease progression received targeted therapy or PD-1 inhibitor in the S-1 and DP group.

The median follow-up time was 19 months (range: 1-66 months). As of May 31, 2020, 55 patients $(76.4 \%)$ in the S-1 group and 73 patients $(70.9 \%)$ in the DP group had died or were followed for more than 3 years. The median OS was not reached in both groups. The 1 - and 3-year survival rates were $72.2 \%$ and $34.7 \%$, respectively in S-1 group, and $75.7 \%$ and $38.8 \%$, respectively in DP group. As shown in Fig. 1B, OS was not significantly different between the two groups $(p=0.422)$.

Because there were more patients in the DP group who did not complete the all cycles of concurrent chemotherapy than in the S-1 group, we further investigated the role on survival. As shown in Fig. 1C-D, patients in the DP group who completed the planned concurrent chemotherapy had longer PFS ( $p=$ $0.011)$ and $O S(p=0.016)$ than those who did not.

\section{Discussion}


In this retrospective study, we compared the clinical safety and efficacy between S-1 and DP as part of CCRT in patients with locally advanced ESCC. Our results showed that compared with DP, S-1 was noninferior in terms of treatment response rate, PFS and OS. However, the incidences of severe treatmentrelated toxicities were significantly lower in S-1 group than in the DP group.

Concurrent chemotherapy with RT has been established as the standard treatment for patients with locally advanced ESCC.[19] The concurrent chemotherapy with PF is most commonly used in clinical

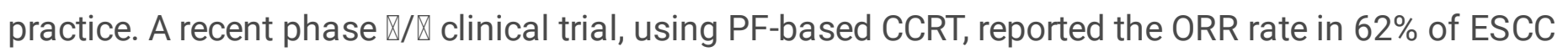
patients and 7.5\% survived for 3-year. In this study, the ORR and the 3-year OS were comparable or even better than those patients treated with PF-based CCRT.[20]

Docetaxel is semi-synthetic taxanes that has radiosensitizing effect through inducing the G2/M cell cycle blockage.[21] Several recent clinical trials of docetaxel plus cisplatin concurrent with RT in locally advanced ESCC have shown promising results and manageable toxicity. In a phase $\otimes$ clinical trial, Shim $\mathrm{Hj}$ evaluated CCRT with weekly DP for advanced ESCC, and showed that this regimen was convenient and well tolerable. This study showed the overall response rate of $85.8 \%, 3$ year PFS of $16.7 \%$,and 3 year OS of $27.8 \%$.[22] In our study, the ORR was $73.8 \%$, and the 3 -year OS was $38.8 \%$ in the DP group, which is

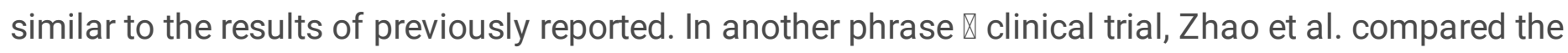
CCRT with either DP or PF in patients with ESCC, and demonstrated a survival advantage from the DP regimen.[23] In contrast, Zhu Y et al. compared with DP with PF in 86 patients with ESCC who received CCRT and demonstrated that OS, PFS, and treatment response were nearly equivalent in both groups. However, the incidence of Grade $\geq 3$ neutropenia was significantly higher in the DP-based treatment (68.9\% vs $19.5 \%) .[24]$

Compared with 5-fluorouracil, S-1, a fourth-generation orally active fluoropyrimidine,exhibits higher anticancer activity and lower side effect. Several studies have evaluated the efficacy of CCRT using S-1 based chemotherapy. In a retrospective analysis of CCRT with S-1 in 68 patients with stage $\mathbb{\nabla}-\mathrm{ESCC}$, the median OS was 25.7 months, and the 1-year, 3-year and 5-year OS rate were $70.6 \%, 41.8 \%$ and $25.9 \%$, respectively.[25] In a prospective study of 30 patients received CCRT with $\mathrm{S}-1\left(70 \mathrm{mg} / \mathrm{m}^{2}\right)$, the 2-years PFS and OS was $40.8 \%$ and $45.1 \%$, respectively.[26] However, no study has directly compared the efficacy and safety of concurrent DP and S-1 regimens combined with RT in ESCC. In this study, the 3-year OS and 3-year PFS in the S-1 group was $34.7 \%$ and $25.0 \%$, respectively. Compared with S-1, DP-based CCRT did not prolong OS and PFS. The non-significant difference in prognosis between the two groups may be due to the poor treatment completion rate in the DP group. In our study, only 73 patients in the DP group completed the concurrent chemotherapy as planned, which was significantly lower than that in the S-1 group $(70.9 \%$ versus $86.1 \%)$. The 3-year PFS and OS were significantly longer for patients who completed the planned concurrent chemotherapy than those who did not. Another reason could partially attribute to the therapy followed the study. More patients in the S-1 group (42.1\% versus $32.0 \%$ ) received targeted therapy or PD-1 inhibitor after tumor progression. 
Severe hematological toxicities are the main reason for treatment interruption or termination. In the present study, the incidence of grade 3-4 hematological toxicities was significantly higher in the DP group than in the S-1 group. The most frequent $\geq$ grade 3 toxicity in DP group was leukopenia, observed approximately in $34.0 \%$ of patients, which was higher than in previous studies.[27] Though analysis, we found that the higher adverse event rate may be explained by the poor general condition of patients and older age before treatment. As most grade 3-4 leukopenia were observed at cycle 3, primary prophylactic granulocyte colony-stimulating factors (G-CSF) should be strongly considered in the management of these patients. In addition, several non-hematological toxicities (nausea and vomiting) were more frequent and severe in the DP group than in the S-1 group, most likely due to the use of highly emetogenic cisplatin. In this study, grade 3-4 adverse events were less observed in the S- 1 group, with a rate of only $22.2 \%$, which is similar to previous study.[25] CCRT with S-1 means a lower incidence of treatment-related toxicities, so this regimen should be more recommended for those who are weak or older.

This study is limited for the short follow-up period and the retrospective design. Therefore, these results need to be validated in prospective study. In addition, the sample size was small and a larger sample would be needed to make these conclusions more robust.

\section{Conclusion}

Our findings suggest that CCRT with S-1 is non-inferior to CCRT with DP in term of treatment response, PFS, and OS. CCRT with S-1 could be a new treatment option for patients with locally advanced ESCC. Considering that potential bias may exist in this study, randomized clinical trials are needed to confirm these findings.

\section{List Of Abbreviations}

CCRT: concurrent chemoradiotherapy; ESCC: esophageal squamous cell carcinoma; OS: overall survival; PF: cisplatin and fluorouracil; DP: docetaxel and cisplatin; RT: radiotherapy; KPS: Karnofsky performance status; 3D-CRT: three-dimensional conformal radiotherapy; IMRT: intensity modulated radiotherapy; CTV: clinical target volume; GTV: gross tumor volume; NCI CTCAE: National Institute Common Terminology Criteria for Adverse Events; RECIST: Response Evaluation Criteria in Solid Tumor; ORR: objective response rate; $C R$ : complete response; PR: partial response; SD: stable disease; PD: progressive disease; PFS: progression free survival.

\section{Declarations}

\section{Ethics approval and consent to participate}

This study was approved by the institutional review board of Huai'an First Hospital. Informed consent was exempted due to the retrospective nature of the study.

\section{Consent for publication}


Informed consent was obtained from all participants for publication.

\section{Availability of data and materials}

All data included in the present study were presented in the main manuscript.

\section{Competing interests}

The authors declare no conflict of interest.

\section{Funding}

This project was supported by the Huai'an Natural Science Research Project (HAB201930).

\section{Authors' contributions}

XLZ and YST conceived and designed the experiments and were responsible for data analysis and writing the manuscript. CHY, WGZ and WWW were responsible for providing the clinical samples. FZJ and YZX were responsible for data collection. All authors read and approved the final manuscript.

\section{Acknowledgments}

The authors acknowledge Juan Tan for his professional statistical analysis.

\section{References}

1. Liu Q, Xia Y, Chen Y, Zhang J, Deng J, Zhao K. A study of concurrent chemoradiotherapy with weekly docetaxel and cisplatin for advanced esophageal squamous cell carcinoma with T4 and/or M1 lymph node metastasis or locoregional recurrence. Radiat Oncol. 2020, 15:75.

2. Zhang H, Yue J, Jiang Z, Zhou R, Xie R, Xu Y, Wu S. CAF-secreted CXCL1 conferred radioresistance by regulating DNA damage response in a ROS-dependent manner in esophageal squamous cell carcinoma. Cell Death Dis. 2017, 8:e2790.

3. Yamashita H, Omori M, Takenaka R, Okuma K, Kobayashi R, Ohtomo K, et al. Involved-field irradiation concurrently combined with nedaplatin/5-fluorouracil for inoperable esophageal cancer on basis of 18FDG-PET scans: A phase II study. Radiother Oncol. 2014, 113:182-187.

4. Honing J, Smit JK, Muijs CT, Burgerhof JGM, de Groot JW, Paardekooper G, et al. A comparison of carboplatin and paclitaxel with cisplatinum and 5-fluorouracil in definitive chemoradiation in esophageal cancer patients. Ann Oncol. 2014, 25:638-643.

5. Cooper JS, Guo MD, Herskovic A, Macdonald JS, Martenson JA Jr, Al-Sarraf M, et al. Chemoradiotherapy of locally advanced esophageal cancer: long-term follow-up of a prospective randomized trial (RTOG 85-01). JAMA.1999;281(17):1623-7.

6. Kim DE, Kim UJ, Choi WY, Kim MY, Kim SH, Kim MJ, et al. Clinical prognostic factors for locally advanced esophageal squamous carcinoma treated after definitive chemoradiotherapy. Cancer Res 
Treat. 2013, 45:276-284.

7. Xi M, Zhang P, Zhang L, Yang YD, Liu SL, Li Y, et al. Comparing docetaxel plus cisplatin versus fluorouracil plus cisplatin in esophageal squamous cell carcinoma treated with neoadjuvant chemoradiotherapy. Jpn J Clin Oncol. 2017, 47:683-689.

8. Bao Y, Liu S, Zhou Q, Cai P, Anfossi S, Li Q, et al. Three-dimensional conformal radiotherapy with concurrent chemotherapy for postoperative recurrence of esophageal squamous cell carcinoma: clinical efficacy and failure pattern. Radiat Oncol. 2013, 8:241.

9. Zhang P, Xi M, Li QQ, Hu YH, Guo X, Zhao L, et al. Concurrent cisplatin and 5-fluorouracil versus concurrent cisplatin and docetaxel with radiotherapy for esophageal squamous cell carcinoma: a propensity score-matched analysis. Oncotarget. 2016;7(28):44686-44694.

10. Pfeiffer P, Qvortrup C, Krogh M, Schoennemann K, Vestermark LW, Jensen HA, et al. S-1 in combination with docetaxel and oxaliplatin in patients with advanced gastro-esophageal adenocarcinoma: two parallel phase 1/2a studies. Acta Oncologica. 2016, 56:46-51.

11. Jeung H-C, Rha SY, Im CK, Shin SJ, Ahn JB, Yang WI, et al. A randomized phase 2 study of docetaxel and S-1 versus docetaxel and cisplatin in advanced gastric cancer with an evaluation of SPARC expression for personalized therapy. Cancer. 2011, 117:2050-2057.

12. Kubota K, Sakai H, Katakami N, Nishio M, Inoue A, Okamoto H, et al. A randomized phase III trial of oral S-1 plus cisplatin versus docetaxel plus cisplatin in Japanese patients with advanced non-smallcell lung cancer: TCOG0701 CATS trial. Ann Oncol. 2015, 26:1401-1408.

13. Hye Ryun Kim, Chang Geo Lee, Eun Chang Choi , Joo Hang Kim, Yoon Woo Koh, Byoung Chul Cho. Induction docetaxel and S-1 followed by concomitant radiotherapy with low-dose daily cisplatin in locally advanced head and neck carcinoma. Head Neck. 2016;38 Suppl 1:E1653-9.

14. Feijiao Ge, Nong Xu, Yuxian Bai, Yi Ba, Yanqiao Zhang, Fei Li, et al. S-1 as Monotherapy or in Combination With Leucovorin as Second-Line Treatment in Gemcitabine-Refractory Advanced Pancreatic Cancer A Randomized, Open-Label, Multicenter, Phase II Study. Oncologist. 2014;19(11):1133-4.

15. Chang H, Shin SK, Cho BC, Lee C-G, Kim CB, Kim DJ, et al. A prospective phase II trial of S-1 and cisplatin-based chemoradiotherapy for locoregionally advanced esophageal cancer. Cancer Chemother Pharmacol. 2014, 73:665-671.

16. Tahara M, Fuse N, Mizusawa J, Sato A, Nihei K, Kanato K, et al. Phase I/II trial of chemoradiotherapy with concurrent S-1 and cisplatin for clinical stage II/III esophageal carcinoma (JCOG 0604). Cancer Sci. 2015, 106:1414-1420.

17. Zhou XL, Zhu WG, Zhu ZJ, Wang WW, Deng X, Tao WJ, et al. Lymphopenia in Esophageal Squamous Cell Carcinoma: Relationship to Malnutrition, Various Disease Parameters, and Response to Concurrent Chemoradiotherapy. Oncologist. 2019, 24:e677-e686.

18. P Therasse, S G Arbuck, E A Eisenhauer, J Wanders, R S Kaplan, L Rubinstein, et al. New Guidelines to Evaluate the Response to Treatment in Solid Tumors. J Natl Cancer Inst. 2000;92(3):205-16. 
19. Yun Chen, Jinjun Ye, Zhengfei Zhu, Weixin Zhao, Jialiang Zhou, et al. Chaoyang Wu, et al. Comparing Paclitaxel Plus Fluorouracil Versus Cisplatin Plus Fluorouracil in Chemoradiotherapy for Locally Advanced Esophageal Squamous Cell Cancer: A Randomized, Multicenter, Phase III Clinical Trial. J Clin Oncol. 2019;37(20):1695-1703.

20. Conroy T, Galais M-P, Raoul J-L, Bouché O, Gourgou-Bourgade S, Douillard J-Y, et al. Definitive chemoradiotherapy with FOLFOX versus fluorouracil and cisplatin in patients with oesophageal cancer (PRODIGE5/ACCORD17): final results of a randomised, phase 2/3 trial. Lancet Oncol. 2014, 15:305-314.

21. Higuchi K, Komori S, Tanabe S, Katada C, Azuma M, Ishiyama H, et al. Definitive chemoradiation therapy with docetaxel, cisplatin, and 5-fluorouracil (DCF-R) in advanced esophageal cancer: a phase 2 trial (KDOG 0501-P2). Int J Radiat Oncol Biol Phys. 2014, 89:872-879.

22. Shim H-J, Kim D-E, Hwang J-E, Bae W-K, Nam T-K, Na K-J, et al. A phase Il study of concurrent chemoradiotherapy with weekly docetaxel and cisplatin in advanced oesophageal cancer. Cancer Chemother Pharmacol. 2012, 70:683-690.

23. Zhao T, Chen $\mathrm{H}$, Zhang T. Docetaxel and cisplatin concurrent with radiotherapy versus 5 -fluorouracil and cisplatin concurrent with radiotherapy in treatment for locally advanced oesophageal squamous cell carcinoma: a randomized clinical study. Med Oncol. 2012, 29:3017-3023.

24. Zhu Y, Zhang W, Li Q, Li Q, Qiu B, Liu H, et al. A Phase II Randomized Controlled Trial: Definitive Concurrent Chemoradiotherapy with Docetaxel Plus Cisplatin versus 5-Fluorouracil plus Cisplatin in Patients with Oesophageal Squamous Cell Carcinoma. J Cancer. 2017, 8:3657-3666.

25. Lv S, Fang M, Yang J, Zhan W, Jia Y, Xu H, et al. Long-term results of definitive concurrent chemoradiotherapy using S-1 in the treatment of geriatric patients with esophageal cancer. Onco Targets Ther. 2016, 9:5389-5397.

26. Ji Y, Du X, Tian Y, Sheng L, Cheng L, Chen Y, et al. A phase II study of S-1 with concurrent radiotherapy in elderly patients with esophageal cancer. Oncotarget. 2017, 8:83022-83029.

27. Hu G, Wang Z, Wang Y, Zhang Q, Tang N, Guo J, et al. Comparison of cisplatinum/paclitaxel with cisplatinum/5-fluorouracil as first-line therapy for nonsurgical locally advanced esophageal squamous cell carcinoma patients. Drug Des Devel Ther. 2016, 10:2129-2136.

\section{Figures}


A

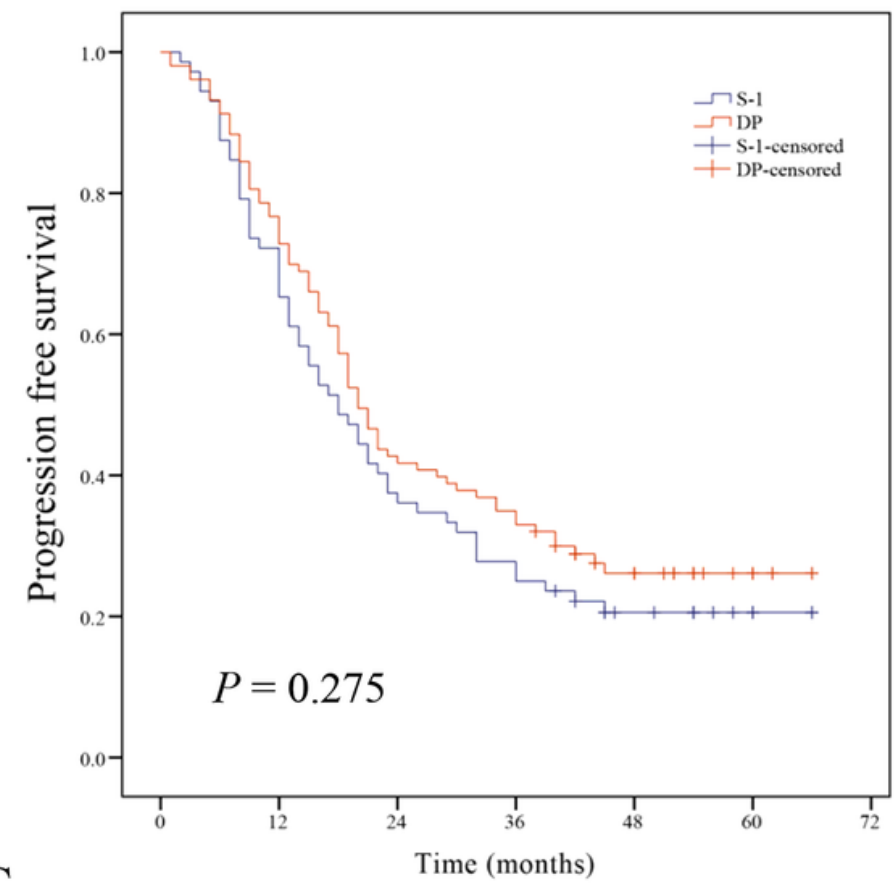

$\mathrm{C}$

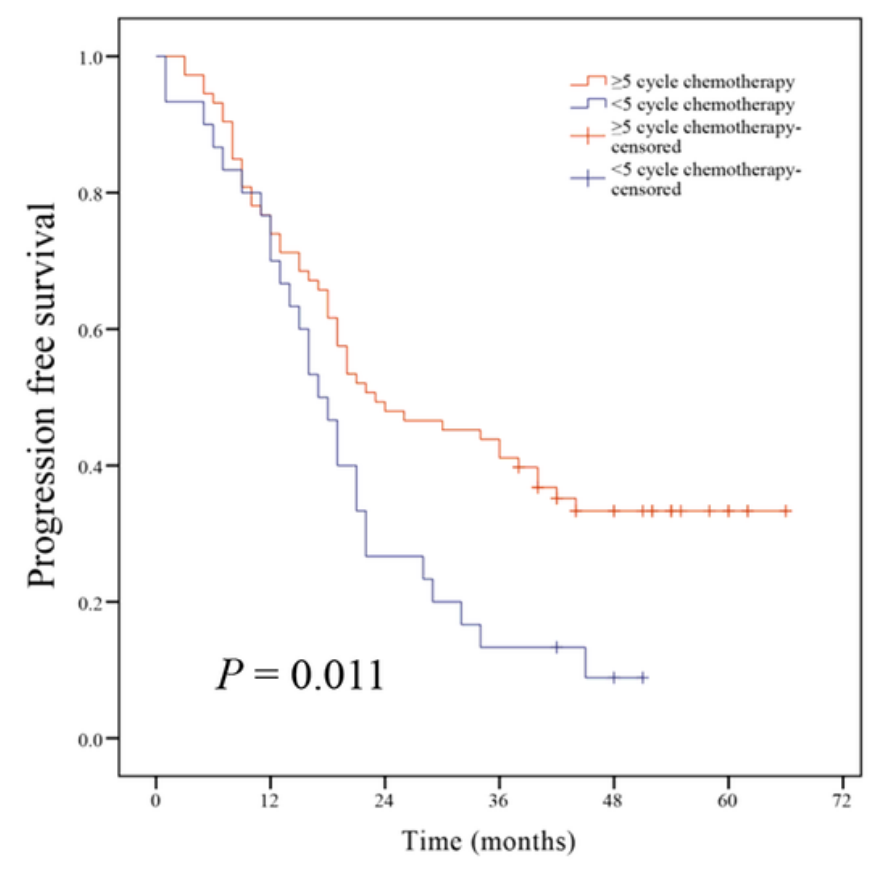

B

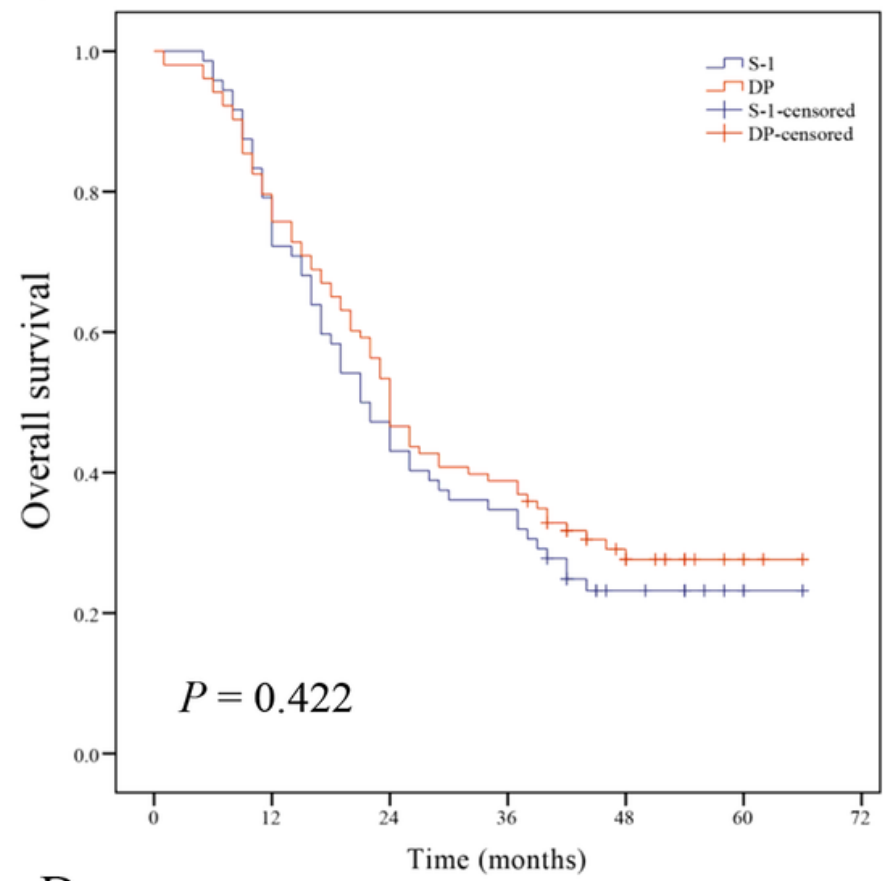

$\mathrm{D}$

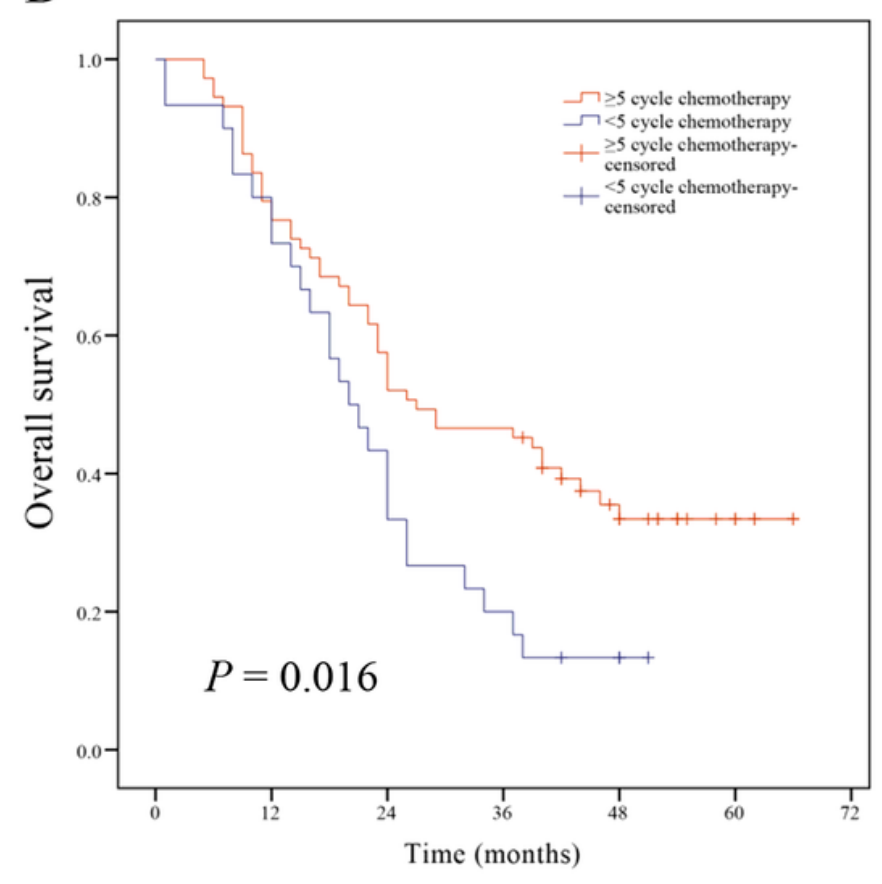

\section{Figure 1}

Kaplan-Meier estimates of progression free survival (A) and overall survival (B) in the S-1 and DP groups. Kaplan-Meier estimates by completeness of concurrent chemotherapy for progression free survival (C) and overall survival (D) using patients in the DP group. 